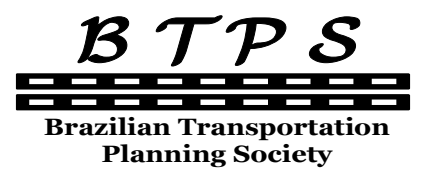

Journal of Transport Literature

Vol. 8, n. 4, pp. 37-59, Oct. 2014

Research Directory
JTL|RELIT

www.journal-of-transport-literature.org ISSN 2238-1031

\title{
Aplicação de novo modelo simulação de fusões ao transporte aéreo
}

[Applying a new merger simulation model to the airline industry]

\author{
Sergio Aquino de Souza* \\ Federal University of Ceará - Brazil
}

Submitted 18 Jun 2013; received in revised form 5 Jul 2013; accepted 18 Oct 2013

\begin{abstract}
Resumo
Este artigo apresenta o Antitrust Mixed Logit Model (AMLM), uma metodologia nova que demonstra como calibrar os parâmetros de um modelo de demanda mixed-logit e simular os efeitos unilaterais decorrentes de uma fusão horizontal. A principal vantagem sobre a versão mais simples que utiliza o modelo logit (Antitrust Logit Model, ALM, desenvolvido por Werden e1994) é a flexibilidade da demanda, o que resulta em elasticidades mais plausíveis e, conseqüentemente, previsões mais plausíveis sobre o efeito de fusões. Ao contrário de abordagens econométricas, o AMLM compartilha com o ALM atributos interessantes para autoridades de defesa da concorrência: requer poucos dados e é rápido de processar. Utilizam-se dados fictícios para ilustrar a operação, principais inputs e outputs do AMLM e comparar os resultados com outros modelos como ALM e o PCAIDS (Proportionally Calibrated Almost Ideal Demand System). Adicionalmente, aplica-se o modelo AMLM às simulações entre várias empresas que compõem o mercado de transporte aéreo de passageiros, incluindo a recente fusão real entre GOL e WEBJET.
\end{abstract}

Palavras-Chave: transporte aéreo, mixed logit, simulação de fusão, defesa da concorrência.

\begin{abstract}
This paper presents the Antitrust Mixed Logit Model (AMLM), a novel methodology that shows how to calibrate the parameters of a mixed-logit demand model and simulate the competitive effects of horizontal mergers. The major advantage over the simpler Logit version (the Antitrust Logit Model, ALM, developed by Werden and Froeb,1994) is flexibility, resulting in more reasonable elasticities and consequently more plausible predictions of merger effects. Moreover, unlike the econometric approaches, the AMLM shares with the ALM the attributes that are particularly appealing to antitrust agencies, given time and data constraints they usually face: low data requirement and high computational speed. The methodology is illustrated with fictitious data to compare the results with other competing models, such as ALM and PCAIDS. Additionally, the model is applied to simulate several mergers in the Brazilian airline industry, including the recent actual merger between GOL and WEBJET merger.
\end{abstract}

Key words: airline industry, mixed logit, merger simulation, competition policy.

*Email: srgdesouz@gmail.com.

\section{Recommended Citation}

DeSouza, Sergio A. (2014) Aplicação de novo modelo simulação de fusões ao transporte aéreo. Journal of Transport Literature, vol. 8, n. 4, pp. 37-59.

- JTL/RELIT is a fully electronic, peer-reviewed, open access, international journal focused on emerging transport markets and published by BPTS - Brazilian Transport Planning Society. Website www.journal-of-transport-literature.org. ISSN 2238-1031.

This paper is downloadable at dx.doi.org/10.1590/2238-1031.jtl.v8n4a2 


\section{Introdução}

Prever os efeitos unilaterais decorrentes de fusões horizontais constitui um dos principais objetivos das agências de Defesa da Concorrência. Comumente, métodos qualitativos são combinados com outros métodos tradicionais para avaliar a potencial perda de bem-estar devido à eliminação da concorrência entre duas ou mais empresas. No entanto, devido ao rápido desenvolvimento de novas ferramentas em organização industrial e empírica a crescente aceitação pelas agências antitruste e tribunais, a aplicação de métodos quantitativos tem crescido significativamente. Entre estes, um dos métodos mais utilizados é a simulação de fusões, que faz previsões quantitativas de alterações nos preços devido à eliminação da rivalidade entre duas empresas baseadas a partir de um modelo estrutural de demanda e oferta.

Há muitas formas de estruturar um modelo de simulação de fusões. No lado da demanda, o produto tem que ser definido como um bem homogêneo ou diferenciado. Por sua vez, do lado da oferta as opções típicas são Bertrand (competição em preços) e Cournot (competição em quantidade). A técnica de simulação é mais freqüentemente aplicada para prever os efeitos concorrenciais de fusões em indústrias caracterizadas por bens diferenciados utilizando o jogo Bertrand. Neste caso, as opções comuns para a demanda são: modelos contínuos, tais como AIDS, linear e log-linear, e modelos de escolha discreta, tais como Logit, Nested logit e Mixed $\operatorname{Logit}^{1}$.

A metodologia empírica para determinar os parâmetros de demanda e oferta, a partir do quais são realizadas as simulações, também apresenta alternativas. De fato, os modelos podem ser calibrados ou estimados econometricamente. A abordagem econométrica normalmente exige a coleta de um conjunto de dados abrangente (preços, quantidades, deslocadores de demanda e custo, além de instrumentos) e, por vezes, os modelos apresentam elevada carga computacional (Berry, Levinsohn e Pakes,1995 e Nevo, 2000 e 2001).

O benefício mais explícito da abordagem econométrica é a possibilidade de avaliar a precisão das estimativas e, portanto, testar os parâmetros (demanda e oferta) e resultados (elasticidades e aumento de preços) com critérios estatísticos bem definidos. Uma alternativa é adicionar

\footnotetext{
${ }^{1}$ Ver Budzinski e Ruhmer (2009) para uma descrição extensa sobre modelos de simulação.
} 
hipóteses ao modelo e informações extras (como por exemplo, um subconjunto da matriz de elasticidades) para recuperar os parâmetros do modelo de forma determinística, ou seja, para calibrar os parâmetros. Neste caso, além da informação externa, somente preços e quantidades (ou fatias de mercado) são necessários e, devido à simplicidade do modelo, o cálculo torna-se mais rápido. A desvantagem da calibragem é não possuir uma forma natural de testar os parâmetros e as previsões de mudança nos preços, além de exigir muita confiança sobre a informação externa. Este problema é atenuado, porém, utilizando a análise de sensibilidade.

Dentro da classe de modelos calibrados, o Antitrust Logit Model (ALM, doravante), desenvolvido por Werden e Froeb (1994), constitui o modelo de referência, pois boa parte dos modelos atuais seguem sua estrutura (demanda de escolha discreta por produtos diferenciados com competição à La Bertrand) e abordagem empírica (calibrar os parâmetros e recalcular os preços após a eventual fusão). No entanto, é notório que o modelo de demanda Logit impõe limitações muito severas sobre as elasticidades-preço (tanto a própria quanto a cruzada), que constituem importantes variáveis econômicos na avaliação dos efeitos de fusões ${ }^{2}$.

Este artigo apresenta o Antitrust Mixed Logit Model (AMLM), uma nova metodologia, cuja principal contribuição é mostrar como calibrar os parâmetros de um modelo de demanda de escolha discreta Mixed Logit. Após este passo, a calibragem do modelo utiliza o conceito de equilíbrio de Bertrand-Nash no contexto de produtos diferenciados a fim de obter os custos marginais e simular os aumentos de preços.

O fator que distingue os dois modelos, AMLM e ALM, reside no lado demanda, elemento essencial na simulação. De fato, a grande vantagem do AMLM sobre a versão Logit (ALM) é a flexibilidade da demanda Mixed Logit, que gera padrões mais razoáveis de substituição entre bens e, conseqüentemente, previsões mais plausíveis sobre os efeitos de uma determinada fusão. Além disso, ao contrário das abordagens econométricas para estimar os parâmetros de uma demanda Mixed Logit (Berry, Levinsohn e Pakes,1995 e Nevo,2000,2001), o AMLM exibe atributos que são particularmente atrativos para agências de Antitruste: baixa exigência de dados e alta velocidade computacional.

\footnotetext{
${ }^{2}$ Este é um problema estrutural do modelo logit, no sentido de que, independentemente da metodologia empírica empregada para descobrir seus parâmetros (calibração ou econometria), o modelo impõe por construção uma matriz de elasticidade inflexível (ou seja, uma matriz com muitos elementos idênticos).
} 
Este artigo está organizado da seguinte forma. A Seção 2 apresenta modelo de demanda Logit. A Seção seguinte introduz o Mixed Logit. Por sua vez, a Seção 4 apresenta o lado da oferta, em que as empresas concorrem à la Bertrand. Na Seção 5, dados fictícios são usados para ilustrar a AMLM. Outra aplicação com dados reais do setor aéreo é apresentada na Seção 6. Finalmente, comentários adicionais podem ser encontrados na última seção.

\section{Calibragem da demanda Logit}

Como ponto de partida, apresenta-se o lado da demanda do $\mathrm{ALM}^{3}$, que propõe a calibração do modelo mais simples de demanda de escolha discreta: Logit. Esse modelo produz soluções analíticas para a demanda e as elasticidades e, portanto, permite destacar de forma clara e simples as idéias básicas do ALM, que servem de inspiração para calibrar o modelo mais flexível apresentado na próxima seção.

Assim como outros modelos de escolha discreta, o Logit assume que a escolha do produto se dá de forma indireta a partir das preferências dos consumidores pelos atributos e não pelo produto em si. Uma vez mapeadas as preferências do consumidor pelos atributos é possível inferir a distribuição de preferências pelos produtos. Os modelos de escolha discreta rompem a relação entre número de produtos e número de parâmetros, facilitando aplicação métodos empíricos, especialmente a calibragem. Não interessa o quão grande seja a quantidade de produtos, o número de parâmetros a ser estimado será o mesmo. Isto é verdade para todos os modelos de escolha discreta, no entanto, este resultado fica bem mais claro no caso do modelo Logit descrito a seguir.

Formalmente, neste modelo os consumidores ordenam suas preferências pelos produtos (ou marcas) de acordo com suas características e preços. Existem $N+1$ escolhas no mercado, $N$ bens internos e um bem de referência (ou bem externo). O consumidor $i$ escolhe a marca $j$, dado o preço $p_{j}$, características não observadas (sintetizadas pelo escalar $\delta_{j}$ ) e as preferências idiossincráticas não observadas $\varepsilon_{i j}$, de acordo com a função de utilidade a seguir:

\footnotetext{
${ }^{3}$ O modelo da oferta é idêntico tanto to ALM quanto AMLM e segue o padrão da literatura: competição com produtos diferenciados à la Bertrand.
} 


$$
u_{i j}=-\alpha p_{j}+\delta_{j}+\varepsilon_{i j}
$$

onde $\alpha$ é um coeficiente que representa a utilidade marginal do consumidor $i$ (ou desutilidade) em relação ao preço. A especificação da demanda se completa com a definição do bem externo, bem substituto aos bens internos ao qual o consumidor atribui utilidade nula, uma normalização típica de modelos de escolha discreta. O consumidor então escolhe o produto que lhe confere maior utilidade, o que corresponde à solução da seguinte maximização, $\operatorname{Max}\left\{u_{i j}, j=0,1, . . N\right\}$, onde $N$ representa o número de bens internos. McFadden (1974, p.3940) mostra que, ao assumir uma distribuição Gumbel (valor extremo) para $\varepsilon_{i j}$ $\left(f\left(\varepsilon_{i j}\right)=\exp \left(-\exp \left(\varepsilon_{i j}\right)\right)\right.$, obtém-se uma forma analítica para a probabilidade de o consumidor $i$ escolher o determinado produto $j$, cuja fórmula é dada por

$$
\sigma_{i j}=\frac{e^{\delta_{j}}}{\sum_{k=0}^{1} e^{\delta_{k}}}
$$

No entanto, como os dados tipicamente disponíveis estão em nível de produto (ou marca), não em nível do consumidor, é preciso obter a probabilidade de escolha do bem $j\left(\sigma_{j}\right)$, que, neste caso, é simples de calcular. Observe que a equação acima não é indexada pelo consumidor $i$, portanto, a probabilidade a probabilidade do produto $j$ ser escolhido é mesma para todo consumidor i e, portanto, a probabilidade (não condicional) de escolha do produto $j$, dada por $\sigma_{j} e ́$ mesma a probabilidade de escolha do produto $j$ pelo consumidor $i$. Portanto $\sigma_{j}=\sigma_{i j}$. Por sua vez, a probabilidade condicionada à escolha de um bem interno $j\left(\sigma_{j l}\right)$, ou seja, a fatia de mercado condicionada ${ }^{4}$ do bem interno $\mathrm{j}\left(s_{j I}\right)$ é dada por

$$
s_{j I}=\sigma_{j I}(\alpha, p, \delta)=\frac{\sigma_{j}(\alpha, p, \delta)}{\sigma_{I}(\alpha, p, \delta)}=\frac{\sigma_{j}(\alpha, p, \delta)}{1-\sigma_{0}(\alpha, p, \delta)}
$$

\footnotetext{
${ }^{4}$ Termo utilizado por Werden and Froeb (2008, p.43) para designar a fatia de mercado dentro do conjunto dos bens internos, sendo, portanto, calculada pela razão entre quantidade do bem $\mathrm{j}$ e a quantidade total dos bens internos.
} 
Onde $\delta$ e $\mathrm{p}$ são vetores vetor $N$-dimensionais definidos, respectivamente por $\delta=\left(\delta_{1}, \delta_{2}, \delta_{3} \ldots \delta_{N}\right)$ e $p=\left(p_{1}, p_{2}, p_{3} \ldots . p_{N}\right) ; \sigma_{I}(\alpha, p, \delta)$ é a probabilidade de escolha do conjunto dos bens internos e $\sigma_{0}(\alpha, p, \delta)$ é a probabilidade de escolher o bem externo.

Como em qualquer modelo de calibração, o ALM acrescenta mais informações ao modelo. Esta informação pode vir de diferentes fontes, tais como documentos de empresas, outros estudos e a opinião de especialistas da indústria. Neste modelo, somente duas elasticidades são suficientes para recuperar os parâmetros de demanda. Normalmente, a elasticidade-preço própria de um dos bens internos e a elasticidade agregada (indústria) compõem o conjunto de informações externas, mas outras combinações podem ser utilizadas, tais como elasticidadespreço próprias para duas mercadorias diferentes ou duas diferentes elasticidades-preço cruzadas.

O Logit implica nas seguintes fórmulas analíticas para a elasticidade agregadas $\eta_{I}$ e elasticidade própria dos preços $\eta_{l l}$ :

$$
\begin{aligned}
& \eta_{I}(\alpha, p, \delta)=-\alpha \bar{p} \sigma_{0} \quad e \\
& \eta_{l l}(\alpha, p, \delta)=-\alpha p_{l}\left[1-\sigma_{l}\right]
\end{aligned}
$$

onde, $\quad \eta_{l l}=\frac{p_{l}}{\sigma_{l}} \frac{\partial \sigma_{l}}{\partial p_{l}}, \quad \eta_{I}=-\left.\frac{\lambda}{S_{I}(\lambda p)} \frac{\partial \sigma_{I}(\lambda p)}{\partial \lambda}\right|_{\lambda=1}$ e $\quad \bar{p}=\sum_{m=1}^{N} \sigma_{m} p_{m}$ é um preço médio ponderado. As equações acima podem ser simplificadas para o seguinte sistema ${ }^{5}$ :

$$
\begin{gathered}
\ln \left[s_{j l}\left(1-\sigma_{0}\right)\right]-\ln \left[\sigma_{0}\right]=-o p_{j}+\delta_{j}, j=1, \ldots, N \\
\left|\eta_{l}\right|=\frac{\left[\alpha \bar{p}\left(1-s_{l I}\right)+\left|\eta_{I}\right| s_{l I}\right] p_{l}}{\bar{p}}
\end{gathered}
$$

onde $\sigma_{0}=\frac{\left|\eta_{I}\right|}{\alpha \bar{p}}$

${ }^{5} \mathrm{O}$ sistema é linear nas incógnitas $(\delta, \alpha)$. 
A calibração da demanda no ALM consiste simplesmente em resolver o sistema de equações acima para o vetor de dimensão $N+1(\alpha, \delta)$, composto pelo escalar $\alpha$ e o vetor $N$ dimensional $\delta=\left(\delta_{1}, \delta_{2}, \delta_{3} \ldots \delta_{N}\right)$, dados os preços $p=\left(p_{1}, p_{2}, p_{3} \ldots p_{N}\right)$, as fatias de mercado condicionais $\left(s_{j I}\right)$, a elasticidade agregada (ou da indústria) $\eta_{I}$ e a elasticidade de um dos bens internos $\eta_{l}$. Este sistema possui solução simples e analítica. Primeiramente, obtêm-se $\alpha$ a partir de equação (7), o que resulta em $\alpha=\frac{\left|\eta_{j}\right| \bar{p}-\left|\eta_{I}\right| s_{j I} p_{j}}{p_{j} \bar{p}\left(1-s_{j I}\right)}$. E, em seguida, uma vez que $\alpha$ é conhecido, completa-se a calibragem com a obtenção dos $\delta_{j}$ 's a partir do seguinte rearranjo da equação (6): $\delta_{j}=\ln \left[s_{j I}\left(1-\sigma_{0}\right)\right]-\ln \left[\sigma_{0}\right]+\alpha p_{j}$.

Note que a calibragem, ao contrário da abordagem econométrica, exige poucas observações sobre preços e quantidades. De fato, o sistema de equações (6) e (7) pode ser resolvido com apenas três produtos, ou até mesmo dois produtos, o que não é verdadeiro para análise econométrica. A calibragem exige, no entanto, que sejam conhecidas duas elasticidades.

Seja qual for a abordagem empírica (econometria ou calibragem), o modelo Logit apresenta séria limitações, pois impõe um matriz de elasticidades pouco flexível com vários valores idênticos, um propriedade pouco desejável, especialmente se o objetivo for simular fusões. Com relação ao grau de substituição entre dois produtos $j$ e $r$, observe que, a partir da elasticidade cruzada $\eta_{j r}=-\alpha p_{r} \sigma_{r}$ calculadas pelo modelo Logit, o aumento da fatia de mercado do produto $j$ decorrente do aumento percentual do preço $\mathrm{p}_{\mathrm{r}}$ depende apenas de $r$. Isto significa que um aumento percentual de $\mathrm{p}_{\mathrm{r}}$ afetará de forma idêntica todos os outros produtos no mercado (competição não localizada). Trata-se de uma propriedade pouco plausível em mercados com produtos diferenciados. Com efeito, uma das motivações principais para realizar estudos de demanda e simulação de fusão é justamente, para cada par de produtos, distinguir entre mais próximos e mais distantes no espectro de produtos.

Esta limitação do Logit é outra forma de manifestação da propriedade de independência de alternativas irrelevantes (IIA), amplamente discutida na literatura (Mcfadden, 1974 e Berry, 1994). A solução mais simples para esta restrição do modelo Logit é oferecida pelo Modelo Logit Agrupado (ou Nested Logit Model). Neste caso, o pesquisador define a priori os agrupamentos (ou segmentos do mercado) e assume que produtos pertencentes ao mesmo 
grupo possuem grau de substituição mais elevado. No tnatnto, o modelo ainda preserva a propriedade IIa para produtos no mesmo grupo. Outra solução, mais geral e mais sofisticada, se refere ao modelo Logit com coeficientes aleatórios, também conhecido como Mixed Logit, onde a utilidade marginal pelos atributos varia entre os consumidores, gerando um matrix de elasticidades flexível, porpriedade bastante desejável para simulação de fusões.

\section{Calibragem da Demanda Mixed Logit}

Esta seção descreve a contribuição fundamental deste trabalho, que consiste em desenvolver uma metodologia para calibrar os parâmetros do modelo de demanda Mixed Logit. É importante notar que esta contribuição é original no universo de modelos calibrados, visto que soluções econométricas já foram encontradas (Berry, Levinsohn e Pakes,1995) a partir da utilização do Método Geral dos Momentos, que exige elevada carga computacional, muitos dados e bons instrumentos. A motivação deste trabalho consiste, portanto, em desenvolver um método empírico simples, que exija poucos dados como ALM, mas que gere padrão mais plausível de substituição entre produtos como e, conseqüentemente, resultados mais próximos dos reais efeitos econômicos de uma fusão.

Formalmente, neste modelo os consumidores ordenam suas preferências pelos produtos (ou marcas) de acordo com suas características e preços. Existem $N+1$ escolhas no mercado, $N$ bens internos e um bem de referência (ou bem externo). O consumidor $i$ escolhe a marca (ou produto) $j$, dado o preço $p_{j}$, características não observadas (sintetizadas pelo escalar $\delta_{j}$ ) e as preferências idiossincráticas não observadas $\varepsilon_{i j}$, de acordo com a função de utilidade a seguir:

$$
u_{i j}=g\left(\alpha, v_{i}\right) p_{j}+\delta_{j}+\varepsilon_{i j}
$$

Onde o coeficiente de preço $g\left(\alpha, v_{i}\right)$ é um coeficiente aleatório que representa a utilidade marginal do consumidor $i$ (ou desutilidade) do preço, que é uma função do parâmetro $\alpha$ e um termo $v_{i}$ específico que varia entre consumidores. Introduzir heterogeneidade no coeficiente de preço é uma extensão natural do caso particular Logit, no qual $v_{i}$ é uma constante, tornando a utilidade marginal em relação a preços idêntica para todos os consumidores. 
O consumidor então escolhe o produto que lhe confere maior utilidade e, ao assumir uma distribuição de valor extremo do tipo II para $\varepsilon_{i j}\left(f\left(\varepsilon_{i j}\right)=\exp \left(-\exp \left(\varepsilon_{i j}\right)\right)\right.$, é possível obter uma forma analítica para a probabilidade de o consumidor $i$ escolher o determinado produto $j$, cuja fórmula é dada pela familiar fórmula logit

$$
\sigma_{i j}\left(\alpha, p, \delta, v_{i}\right)=\frac{\exp \left(g\left(\alpha, v_{i}\right) p_{j}+\delta_{j}\right)}{1+\sum_{m=1}^{N} \exp \left(g\left(\alpha, v_{i}\right) p_{m}+\delta_{m}\right)}
$$

No entanto, como os dados tipicamente disponíveis estão em nível de produto (ou marca), não em nível do consumidor, é preciso obter a probabilidade de escolha do bem $j\left(\sigma_{j}\right)$, que é dada pelo valor esperado de por $\sigma_{i j}$ em relação à distribuição de $v_{i}$.

$$
\sigma_{j}(\alpha, p, \delta)=E_{v}\left[\sigma_{i j}\left(\alpha, p, \delta, v_{i}\right)\right]=\int \sigma_{i j}\left(\alpha, p, \delta, v_{i}\right) d F(v)
$$

Note que esta integral é trivial no Logit, pois $\sigma_{j}=\sigma_{i j}$, o que não é o caso na equação (10), descrita acima. Por sua vez, a probabilidade condicionada à escolha de um bem interno $\mathrm{j}$ $\left(\sigma_{j l}\right)$, ou seja, a fatia de mercado condicionada do bem interno $\mathrm{j}\left(s_{j I}\right)$ é dada por

$$
s_{j I}=\sigma_{j I}(\alpha, p, \delta)=\frac{\sigma_{j}(\alpha, p, \delta)}{\sigma_{I}(\alpha, p, \delta)}
$$

onde $\sigma_{I}$ é a probabilidade de uma das mercadorias internas ser escolhida.

Para o modelo de demanda Mixed Logit, a elasticidade-preço para um determinado bem $l$ é dada por

$$
\eta_{l l}(\alpha, p, \delta)=\frac{p_{l}}{s_{l I} . \sigma_{I}} E_{v}\left[g\left(\alpha, v_{i}\right) . \sigma_{i l}\left(\alpha, p, \delta, v_{i}\right)\left(1-\sigma_{i l}\left(\alpha, p, \delta, v_{i}\right)\right]\right.
$$

Por sua vez, a elasticidade da demanda agregada $\eta_{I}$ (do conjunto formado pelos bens internos), também conhecida como elasticidade da indústria, é dada por

$$
\eta_{I}(\alpha, p, \delta)=\frac{E_{v}\left[g\left(\alpha, v_{i}\right) \cdot P_{i}\left(\alpha, p, \delta, v_{i}\right) \cdot \sigma_{i 0}\left(\alpha, p, \delta, v_{i}\right)\right]}{\sigma_{I}}
$$


onde $P_{i}=\sum_{m=1}^{N}\left(\sigma_{i m} \cdot p_{m}\right)$ e $\sigma_{i 0}\left(\alpha, p, \delta, v_{i}\right)=\frac{1}{1+\sum_{m=1}^{N} \exp \left(g\left(\alpha, v_{i}\right) p_{m}+\delta_{m}\right)}$ é a probabilidade

do consumidor $i$ escolher o produto externo.

Note que o sistema de equações formado por (11), (12) e (13) pode ser reescrito da seguinte forma:

$$
\begin{gathered}
s_{j I}=\frac{\left|\eta_{I}\right| \cdot \sigma_{j}(\alpha, p, \delta)}{E_{v}\left[\left|g\left(\alpha, v_{i}\right)\right| \cdot P_{i}\left(\alpha, p, \delta, v_{i}\right) \cdot \sigma_{i 0}\left(\alpha, p, \delta, v_{i}\right)\right]}, j=1, \ldots, N \\
\left|\eta_{l l}\right|=\frac{\left|\eta_{I}\right| p_{l}}{s_{l I}} \frac{E_{v}\left[\left|g\left(\alpha, v_{i}\right)\right| \cdot \sigma_{i l}\left(\alpha, p, \delta, v_{i}\right)\left(1-\sigma_{i l}\left(\alpha, p, \delta, v_{i}\right)\right]\right.}{E_{v}\left[\left|g\left(\alpha, v_{i}\right)\right| \cdot P_{i}\left(\alpha, p, \delta, v_{i}\right) \cdot \sigma_{i 0}\left(\alpha, p, \delta, v_{i}\right)\right]}
\end{gathered}
$$

Para realizar a calibragem assume-se que o analista (ou pesquisador) possui o seguinte conjunto de informações: fatias de mercado $s_{j I}$, vetor de preços $p$, distribuição do termo do consumidor específico $v_{i}$, elasticidade agregada $\eta_{I}$ e a elasticidade de um bem $\eta_{l l}$. Desta forma, o sistema chave da metodologia, formado pelas $\mathrm{N}+1$ equações (14) e (15), permite identificar as $N+1$ incógnitas (vetor $\mathrm{N}$-dimensional $\delta$ e o escalar $\alpha)^{6}$, o que completa a calibragem dos parâmetros da demanda que servirão como inputs para a simulação de mudanças de propriedades dos produtos no lado da oferta. Note que apesar, de exigir informações extras sobre duas elasticidades, os parâmetros da demanda do AMLM podem ser determinados com um pequeno conjunto de dados sobre preços e quantidades (fatias). De fato, como no exemplo a ser apresentado na seção 5, é possível determinar parâmetros da demanda e simular fusões com apenas quatro produtos, algo infactível no contexto da abordagem econométrica.

\footnotetext{
${ }^{6} \mathrm{Se} \alpha$ fosse um vetor de dimensão maior que um, e não um escalar como assumido aqui o sistema seria, certamente, sub identificado. Por esta razão, postula-se um modelo Mixed Logit com apenas um coeficiente aleatório com apenas um parâmetro. Se este é um modelo plausível é em grande parte uma questão empírica. Observe também que $\alpha$ é determinista e, portanto, não possui desvio padrão. $\mathrm{O}$ modelo pode ser facilmente estendido para acomodar distribuição mais flexível adicionando outra elasticidade e, conseqüentemente, outra equação para o sistema de calibração.
} 


\section{Oferta}

Determinar os parâmetros de demanda não é suficiente para realizar a simulação de fusão, é preciso modelar o lado da oferta. Este trabalho segue o modelo de Bertrand comumente adotado na literatura: empresas escolhem os preços dos produtos (diferenciados) simultaneamente em um jogo de um só estágio.

Em primeiro lugar, assume-se que cada firma $f$ produz um subconjunto $F_{f}$ dos bens ofertados neste mercado. Em competição à la Bertrand, pode-se mostrar que o preço do produto $j$ produzido pela empresa $f$ a um custo marginal (constante) $c_{j}$ deve satisfazer a seguinte equação

$$
\sigma_{j}+\sum_{r \in F_{f}}\left(p_{r}-c_{r}\right) \frac{\partial \sigma_{r}}{\partial p_{j}}=0 \quad, \quad j=1,2, \ldots, N
$$

$\mathrm{Ou}$, de forma equivalente,

$$
\sigma-(\Omega \Delta[p-c])=0
$$

onde $\sigma, p$ e $c$ são vetores $\mathrm{Nx} 1$ reunindo $\sigma_{j}$ 's, os preços e os custos marginais, respectivamente. Além disso, $\Delta \mathrm{e} \Omega$ são matrizes $N x N$ cujo o elemento característico $(j, r)$ é definido como segue

$$
\begin{aligned}
& \Delta_{j r}=-\frac{\partial \sigma_{r}}{\partial p_{j}} \mathrm{e} \\
& \Omega_{j r} \begin{cases}1 & \text { se r e } j \text { são produzidos pela mesma firma } \\
0 & \text { caso contrário. }\end{cases}
\end{aligned}
$$

A decisão de preços dos bens externos é exógena por hipótese e, portanto, não há interação estratégica com a decisão de preços dos bens internos. Note que (17) é flexível o suficiente para acomodar diferentes estruturas de mercado. A estrutura mais simples é aquela formada por empresas monoproduto, que produzem apenas uma variedade. Outra forma de organização, mais comum, é formada por empresas multiproduto, onde cada firma oferta 
vários produtos diferentes. Um terceiro exemplo é o monopólio ou cartel, onde uma empresa (ou associação) organiza a produção de todas as variedades disponíveis no mercado.

Uma suposição chave em todos os modelos de simulação de fusão consiste em assumir que os preços observados anteriores à operação de concentração horizontal também são gerados pelo resultado de um equilíbrio de Bertrand. Portanto, a equação (17) aplicada aos preços observados antes da fusão é dada por

$$
\sigma\left(p^{p r e}\right)-\left(\Omega^{p r e} \Delta\left(p^{p r e}\right)\left[p^{p r e}-c\right]\right)=0
$$

Observe que $p^{\text {pre }}$ representa o vetor de preços pré-fusão e que $\Omega^{\text {pre }}$ é construído a partir da estrutura de propriedade pré-fusão. Desta forma, a equação (18) permite obter a seguinte fórmula analítica para os custos marginais:

$$
c=p^{\text {pre }}-\left[\left(\Omega^{\text {pre }} \Delta\left(p^{\text {pre }}\right)\right)^{-1} \sigma\left(p^{\text {pre }}\right)\right]
$$

Onde c é um vetor $\mathrm{N}$-dimensional que coleta os custos marginais dos $\mathrm{N}$ produtos ofertados. Uma vez de posse dos parâmetros de demanda e oferta $(\alpha, \delta$ e $c)$ é possível calcular os preços de equilíbrio resultantes da nova estrutura de propriedade decorrente da fusão. De fato, os preços previstos pós-fusão ( $p^{\text {post }}$ ) constituem a solução do seguinte sistema de equações:

$$
\sigma\left(p^{\text {post }}\right)-\left(\Omega^{\text {post }} \Delta\left(p^{\text {post }}\right)\left[p^{\text {post }}-c\right]\right)=0
$$

Onde $\Omega^{\text {post }}$ é construído a partir da estrutura de propriedade pós-fusão. 


\section{Exemplos e outros métodos comparáveis}

Nesta seção, utilizam-se dados fictícios para ilustrar a operação, principais inputs e outputs do AMLM e comparar os resultados com outros modelos como ALM e o PCAIDS (Proportionally Calibrated Almost Ideal Demand System). O ALM é a principal referência de comparação, visto que pertence à mesma classe de modelos de escolha discreta e constitui um caso particular do AMLM. No entanto, comenta-se também brevemente sobre outro modelo de calibragem bastante conhecido, o PCAIDS, desenvolvido por Epstein e Rubinfeld (2002), que modelam as preferências dos consumidores baseadas em um modelo de demanda contínua.

O mercado fictício possui 4 marcas (ou produtos), denominadas, A, B, C e D, onde cada uma é produzida por uma empresa diferente, e apresentam fatias de mercado de 40\%, 35\%, 15\% e $10 \%$, respectivamente. $\mathrm{O}$ preço do bem $\mathrm{A}$ é de 9 unidades monetárias, enquanto $\mathrm{B}, \mathrm{C}$ e $\mathrm{D}$ são vendidos por 6, 5 e 3 unidades monetárias, respectivamente. Além disso, segue-se Petrin (2002) e parametriza-se a utilidade marginal do consumidor em relação a preços de acordo com a forma funcional dada por $g\left(\alpha, v_{i}\right)=-\alpha v_{i}$, em que o termo $v_{i}$ segue uma distribuição qui-quadrado com 3 graus de liberdade. Adicionalmente, para executar o AMLM, são necessárias duas elasticidades. Atribui-se -1 para a elasticidade agregada (ou indústria) e -2 para elasticidade-preço da primeira marca.

O primeiro passo do AMLM é a calibração do sistema de demanda Mixed Logit que consiste em resolver o sistema de cinco equações para o vetor de 5 incógnitas $\left(\alpha, \delta_{1}, \delta_{2}, \delta_{3}, \delta_{4}\right)$, de acordo com o sistema formado por (14) e (15). Encontram-se os valores $\alpha=0.489$, para o parâmetro que compõe o coeficiente aleatório de preço $g\left(\alpha, v_{i}\right)$, e $\delta=(5.343,3.921,2.504,0.663)$, para o vetor que reúne os $\delta_{j}{ }^{\prime} s$.

Observe que $\delta_{j}$ pode se interpretado como qualidade do produto $j$, pois captura atributos, exceto preço, que determinam a utilidade do consumidor. A marca A possui a maior qualidade (5,343), D a menor (0,663), enquanto B e C assumem valores intermediários. Este exemplo simples mostra que os resultados do modelo são consistentes com o que é qualitativamente sugerido pelos dados em um mercado com bens diferenciados. Deve-se 
esperar que os consumidores percebam a marca A como produto superior, pois apesar de ser o mais caro, detém a maior fatia de mercado (40\%). Pelo mesmo motivo, deve-se esperar que a marca D seja a menos desejada pelos consumidores, pois, apesar de ter os preços mais baixos, captura a menor fração do mercado (10\%). Por sua vez, para as marcas B e C, que valores intermediários para preços e fatias de mercado, devem-se esperar valores intermediários para o índice de qualidade.

Outro resultado interessante da calibração da demanda é a matriz de elasticidade. Com os valores calibrados para $\left(\alpha, \delta_{1}, \delta_{2}, \delta_{3}, \delta_{4}\right)$ pode-se usar o modelo e os dados novamente para calcular a elasticidade-preço própria para qualquer bem $j$ a partir da equação:

$$
\eta_{j j}(\alpha, p, \delta)=\frac{p_{j}}{s_{j I} \cdot \sigma_{I}} E_{v}\left[g\left(\alpha, v_{i}\right) \cdot \sigma_{i j}\left(\alpha, p, \delta, v_{i}\right)\left(1-\sigma_{i j}\left(\alpha, p, \delta, v_{i}\right)\right]\right.
$$

Por sua vez, a elasticidade-preço cruzada da demanda de qualquer bem $j$ em relação ao preço de qualquer outro bem $r$ é dada por

$$
\eta_{j r}=\frac{p_{r}}{s_{j I} \cdot \sigma_{I}} E_{v}\left[g\left(\alpha, v_{i}\right) \cdot \sigma_{i r}\left(\alpha, p, \delta, v_{i}\right)\left(\sigma_{i j}\left(\alpha, p, \delta, v_{i}\right)\right]\right.
$$

Aplicando estas fórmulas para este exemplo em particular encontra-se uma matriz 4x4 com os valores dispostos na Tabela 1 .

\section{Tabela 1 - Matriz de Elasticidades AMLM}

\begin{tabular}{lcccc}
\hline \multicolumn{5}{c}{ Em relação a aumento \% de } \\
\hline Elast. do & $\mathrm{P}_{\mathrm{A}}$ & $\mathrm{P}_{\mathrm{B}}$ & $\mathrm{P}_{\mathrm{C}}$ & $\mathrm{P}_{\mathrm{D}}$ \\
Produto & & & & \\
$\mathrm{A}$ & -2.00 & 0.60 & 0.19 & 0.05 \\
$\mathrm{~B}$ & 1.03 & -2.39 & 0.28 & 0.10 \\
$\mathrm{C}$ & 0.94 & 0.80 & -2.75 & 0.12 \\
$\mathrm{D}$ & 0.65 & 0.72 & 0.30 & -2.36 \\
\hline Fonte: Elaboração do autor. & & &
\end{tabular}

A matriz acima (Tabela 1) destaca a principal vantagem de utilizar um sistema de demanda flexível, como no modelo AMLM, que deixa espaço para os dados para determinarem o padrão de substituição entre as marcas. Ao contrário do modelo Logit, que impõe, por construção, que um aumento de preço de um bem $j$ terá o mesmo efeito proporcional na 
demanda de qualquer outro substituto conforme discussão na seção 2. Isso também pode ser verificado empiricamente ao aplicar o modelo ALM para calibrar a demanda utilizando os mesmos dados do AMLM.

\section{Tabela 2 - Matriz de Elasticidades ALM}

\begin{tabular}{|c|c|c|c|c|}
\hline & \multicolumn{4}{|c|}{ Em relação a aumento \% de } \\
\hline $\begin{array}{ll}\text { Elast. } & \text { do } \\
\text { Produto } & \end{array}$ & $\mathrm{P}_{\mathrm{A}}$ & $\mathrm{P}_{\mathrm{B}}$ & $\mathrm{P}_{\mathrm{C}}$ & $\mathrm{P}_{\mathrm{D}}$ \\
\hline A & -2.00 & 0.25 & 0.09 & 0.03 \\
\hline B & 0.44 & -1.37 & 0.09 & 0.03 \\
\hline C & 0.44 & 0.25 & -1.26 & 0.03 \\
\hline $\mathrm{D}$ & 0.44 & 0.25 & 0.09 & -0.77 \\
\hline
\end{tabular}

Note que, se $\mathrm{P}_{\mathrm{A}}$ aumenta em $10 \%$, as demandas de todos os outros bens respondem de maneira uniforme: cada marca verifica aumento de demanda de 4,44\% (ver Coluna 1 da Tabela 2). O mesmo resultado uniforme pode ser encontrado nas outras colunas.

O mesmo padrão de substituição restritivo é encontrado em outra metodologia bem conhecida de calibração: o PCAIDS. Este modelo é muito atrativo em termos operacionais, pois é simples e requer um conjunto muito pequeno de informação: duas elasticidades e fatias de mercado baseadas nas receitas (faturamento), nem mesmo preços são necessários. No entanto, esta simplicidade tem um custo. A fim de calibrar o sistema de demanda com um conjunto limitado de informações, os autores têm de impor uma suposição de proporcionalidade que remove o principal atributo principal de um modelo de demanda contínua ${ }^{7}$ : a flexibilidade da matriz de elasticidade. De fato, como no ALM, o PCAIDS gera uma matriz de elasticidades rígida, com vários elementos idênticos. Outra versão deste modelo, o PCAIDS com ninhos (Epstein e Rubinfeld, 2004), gera melhores resultados do ponto de vista de flexibilidade, mas ainda mostra valores idênticos para as elasticidades-preço cruzada dentro dos ninhos.

De posse dos parâmetros da demanda, procede-se ao cálculo do principal resultado da simulação de fusão: as previsões de aumentos de preços. São simuladas todas as fusões possíveis entre duas empresas. Inicia-se com uma análise detalhada da fusão entre as empresas que produzem os bens A e B, para a qual o AMLM gera os seguintes resultados:

\footnotetext{
${ }^{7}$ O modelo PCAIDS baseia-se no modelo de demanda contínua conhecido como Almost Ideal Demand System (AIDS) que possui matrix flexível, porém apresenta quantidade elevada de parâmetros.
} 
Tabela 3 - Aumento de Preços - Fusão A-B

\begin{tabular}{|c|c|c|c|c|}
\hline $\begin{array}{l}\text { Firmas } \\
\text { na fusão }\end{array}$ & envolvidas & Preços pós-fusão & Preços pré-fusão & Variação (\%) \\
\hline & A & 12.36 & 9 & 37.44 \\
\hline & B & 9.31 & 6 & 55.21 \\
\hline & $\mathrm{C}$ & 5.44 & 5 & 8.91 \\
\hline & D & 3.25 & 3 & 8.57 \\
\hline
\end{tabular}

Fonte: Elaboração do autor.

Em média, os preços (ponderados pelas fatias de mercado) após a fusão são 23,16\% superiores. Observe também a partir da Tabela 3 que todas as marcas são mais caras após a fusão - um típico resultado de simulação de fusão sem ganhos de eficiência- conforme hipótese implícita neste exercício - e que as marcas que exibem a maior variação de preços (A com um aumento de $34,4 \%$ e B com 55,21\%) são aquelas envolvidas na fusão. O mesmo padrão é encontrado para todas as outras fusões, conforme demonstrado na Tabela 4. Por exemplo, a fusão entre A e C causa um aumento de 6,82\% nos preços médios, com estas marcas mostrando os maiores aumentos de preço. Observe, no entanto, que os efeitos anticompetitivos (aumento de preços) são inferiores àqueles obtidos na fusão A-B. Resultados quantitativamente semelhantes podem ser encontrados para a fusão B-C, o que resultará em preços médios 5,42\% maiores após a criação da nova empresa. Efeitos menores são encontrados para as outras fusões: aumento do preço médio de $3,27 \%$ tanto para as fusões AD e B-D e $1,38 \%$ para a fusão C-D.

Tabela 4 - Resultados da Simulação

\begin{tabular}{lccccc}
\hline $\begin{array}{l}\text { Firmas } \\
\text { envolvidas } \\
\text { na fusão }\end{array}$ & $\begin{array}{c}\text { Preços pós- } \\
\text { fusão }\end{array}$ & Preços pré-fusão & $\begin{array}{c}\text { Aumento no } \\
\text { preço médio } \\
(\%)\end{array}$ & $\begin{array}{c}\text { Maior } \\
\text { aumento de } \\
\text { preços(\%) }\end{array}$ & $\begin{array}{c}\text { Marcas com } \\
\text { maiores aumentos }\end{array}$ \\
\hline & & & & & \\
A/B & 8.313 & 6.75 & 23.16 & 55.21 & $A, B$ \\
A/C & 7.211 & 6.75 & 6.82 & 31.191 & $A, C$ \\
A/D & 6.970 & 6.75 & 3.27 & 20.927 & $A, D$ \\
B/C & 7.116 & 6.75 & 5.42 & 17.092 & $B, C$ \\
B/D & 6.970 & 6.75 & 3.27 & 17.505 & $B, D$ \\
C/D & 6.844 & 6.75 & 1.39 & 5.744 & $D, C$ \\
& & & & & \\
\hline
\end{tabular}

Fonte: Elaboração do autor 
Como em outros modelos baseados na calibração, uma forma de avaliar a confiança nos resultados é a realização de análise de robustez em relação à informação passível de maior discussão: o conjunto de elasticidades que o analista impõe ao modelo. Logo, a análise de sensibilidade baseia-se na execução do mesmo modelo com elasticidades diferentes, mas mantém todo o resto igual. Toma-se o conjunto $\{-0,5,-1,-1,5\}$ para a elasticidade da indústria e $\{-2,-2,5,-3\}$ para a elasticidade-preço do produto A e seleciona-se o aumento médio dos preços como o resultado representativo da simulação de fusão entre as marcas A e B.

Tabela 5 - Análise de Sensibilidade-fusão A-B

\begin{tabular}{lccc}
\hline & \multicolumn{3}{c}{ Elasticidade Própria } \\
\hline Elast. da Ind. & -2 & -2.5 & -3 \\
-0.5 & 20.58 & 11.92 & 5.35 \\
-1 & 23.16 & 15.21 & 8.38 \\
-1.5 & 14.52 & 13.90 & 10.25 \\
\hline Fonte: Elaboração do autor
\end{tabular}

A análise de sensibilidade (Tabela 5) mostra as previsões de preços que variam de 5,36\% a $23,16 \%$, o que é aparentemente um intervalo grande, mas os resultados ainda revelam-se úteis uma vez que, mesmo no melhor cenário possível para as partes envolvidas na fusão, os preços apresentam uma considerável aumento $(5,36 \%)$, levantando preocupações sobre os danos à concorrência decorrentes da fusão.

\section{Aplicações ao setor aéreo}

Esta seção aplica o modelo AMLM a dados bem recentes do mercado de transporte aéreo de passageiros no ano de 2010, publicamente disponíveis no sítio da Agência Nacional de Aviação Civil - ANAC. A Tabela 6 abaixo mostra a distribuição do mercado nacional em 2010 a partir de dados de passageiros por quilômetro transportado pago. 
Tabela 6 - Participação e Preços no Mercado de Transp. Aéreo de Passageiros - 2010

\begin{tabular}{ccc}
\hline & Participação (\%) & Preço* (em R\$) \\
\hline TAM & 42,63 & 0,208 \\
GOL & 39,41 & 0,234 \\
AZUL & 6,06 & 0,216 \\
WEBJET & 5,86 & 0,178 \\
Outras & 6,94 & ---- \\
\hline
\end{tabular}

* Preço calculado pelo yield, preço pago por passageiro em um km percorrido

Fonte: Agência Nacional de Aviação Civil - ANAC

Trata-se de um mercado bastante concentrado com as duas maiores empresas apresentando um domínio de $82,04 \%$ do mercado e as quatro maiores com a quase a totalidade do mercado (93,96\%). O índice de concentração medido pelo HHI=3478 também reforça esta observação e a conclusão de que concentrações adicionais são potencialmente preocupantes do ponto de vista da defesa da concorrência. Os preços, medidos em reais que a empresa cobra para transportar um passageiro por um quilômetro, apresentam valores similares para TAM, GOL e AZUL, em torno de 21 centavos e um patamar inferior de 17,8 centavos para a WEBJET.

Como os dados são agregados nacionalmente, conclusões, análises e simulações são preliminares $^{8}$ e fariam parte de estágio inicial de um longo processo de avaliação de uma determinada fusão por parte da autoridade de defesa da concorrência.

A simulação de fusões e, em particular, aquela que utiliza o AMLM, pode ser muito útil nesta avaliação preliminar, pois permite identificar em termos quantitativos o potencial aumento de preços decorrente de determinada concentração horizontal. Como os dados são bem recentes uma escolha natural para ilustrar a metodologia AMLM consiste na simulação da fusão entre GOL e WEBJET. Dada a presença limitada de atores relevantes no setor, é possível simular e comparar a fusão GOL-WEBJET com todas as outras cinco possíveis fusões entre duas empresas.

Todas as simulações realizadas nesta seção seguem a mesma parametrização utilizada anteriormente, ou seja, a utilidade marginal do consumidor em relação a preços é definida por

\footnotetext{
${ }^{8}$ Estudos e análises no setor tipicamente consideram como mercados cada rota, como Rio e São Paulo por exemplo. No entanto, estes dados não estão publicamente disponíveis.
} 
$g\left(\alpha, v_{i}\right)=-\alpha v_{i}$ e o termo $v_{i}$ segue uma distribuição qui-quadrado com 3 graus de liberdade. Além de preços e fatias de mercado o AMLM necessita de dois valores para as elasticidades. Utiliza-se -1 para a elasticidade da indústria, valor normalmente utilizado em modelos calibrados (Epstein e Rubinfeld, 2002,2004) e -2 para a elasticidade própria da TAM, valor encontrado em outros estudos do setor aéreo ${ }^{9}$.

Tabela 7 - Aumento de Preços da Fusão GOL-WEBJET

\begin{tabular}{cccc}
\hline & & & \\
\hline Empresa & Preços pós-fusão & Preços pre-fusão & Variação (\%) \\
TAM & 0,210 & 0,208 & 1.05 \\
GOL & 0,240 & 0,234 & 2.58 \\
AZUL & 0,216 & 0,216 & 0.00 \\
WEBJET & 0,226 & 0,178 & 27.37 \\
\hline
\end{tabular}

Fonte: Elaboração do autor a partir dos resultados do modelo AMLM

Destaca-se na Tabela 7 o aumento expressivo de preços da WEBJET $(27,7 \%)$ e um conseqüente alinhamento de seus preços com as outras marcas. No entanto, o preço médio (ponderado pelas fatias de mercado) aumenta em apenas 2,8\%. Ainda de acordo com a simulação, a GOL eleva de forma moderada seus preços, enquanto que as empresas não envolvidas na fusão apresentam aumentos marginais nos preços, $1,05 \%$ no caso da TAM, ou nulos, como no caso da AZUL.

Os resultados sugerem que o aumento médio nos preços é uma variável pouco representativa dos efeitos anti-competitivos da fusão, pois a distribuição destes efeitos é muito heterogênea entre os bens. Recomenda-se, portanto, utilizar a maior variação $(27,37 \%)$ para concluir, a partir da simulação, que a operação gera significativas preocupações anticoncorrenciais, merecendo uma profunda análise sobre outros fatores que possam vir a mitigar o potencial aumento de preços da WEBJET como a possibilidade, não contemplada nos modelos de simulação, mas consagrada no análise antitruste, de entrada de novas empresas.

Após realizar todas as outras possíveis fusões (Tabela 8) identifica-se um padrão semelhante para as fusões entre uma empresa grande e uma empresa pequena: as operações TAM-AZUL,

\footnotetext{
${ }^{9}$ Note que não necessariamente são utilizadas elasticidades para uma das empresas envolvida na fusão. Neste caso a escolha da TAM deveu-se a consistência de valores em torno de -2, encontrados em vários estudos diferentes (Vassalo,2010 e Ferreira,2011)
} 
TAM-WEB e GOL-AZUL. De fato, encontram aumentos significativos para a empresa menor, pouco superior a $27 \%$, e variação pequena no preço médio, em torno de $3 \%$. Destacam-se ainda as outras duas situações extremas. A primeira envolve a fusão entre as duas maiores empresas TAM e GOL, gerando elevados aumentos tanto no preço médio $(35,77 \%)$ quanto em umas das marcas envolvidas na fusão (51,59\%). A situação oposta é encontrada na simulação da fusão da AZUL com a WEBJET- as duas menores empresas com participação minimamente relevante no setor-, com efeitos pouco significativos sobre preços (aumento de apenas 0,4\% em média e de $2,35 \%$ para a WEBJET).

Tabela 8 - Resultados de todas as Simulações

\begin{tabular}{lccccc}
\hline $\begin{array}{l}\text { Firmas } \\
\text { envolvidas } \\
\text { na fusão }\end{array}$ & $\begin{array}{c}\text { Preço médio } \\
\text { pós-fusão }\end{array}$ & $\begin{array}{c}\text { Preço médio } \\
\text { pré-fusão }\end{array}$ & $\begin{array}{c}\text { Aumento no } \\
\text { preço médio } \\
(\%)\end{array}$ & $\begin{array}{c}\text { Maior } \\
\text { aumento de } \\
\text { preços(\%) }\end{array}$ & $\begin{array}{c}\text { Marca com } \\
\text { maior aumento }\end{array}$ \\
\hline TAM/GOL & 0,2954 & 0,218 & 35,77 & 51,39 & GOL \\
TAM/AZUL & 0,2242 & 0,218 & 3,03 & 27,82 & AZUL \\
TAM/WEB & 0,2238 & 0,218 & 2,87 & 27,13 & WEB \\
GOL/AZUL & 0,2243 & 0,218 & 3,09 & 27,36 & AZUL \\
AZUL/WEB & 0,2185 & 0,218 & 0,40 & 2,35 & WEB \\
\hline
\end{tabular}

Fonte: Elaboração do Autor

Como todo modelo de simulação de fusões, o AMLM impõe uma série de hipótesesinformação sobre elasticidades, modelo de Bertrand com produtos diferenciados, forma funcional da demanda, distribuição dos coeficientes aleatórios, modelo estático, entre outrascom o objetivo de simplificar a análise e ganhar capacidade analítica. Muitas destas hipóteses servem para obter (identificar) os parâmetros de demanda e oferta, não sendo, portanto, testáveis, especialmente no caso de modelos de calibragem, onde não é possível calcular variâncias. No entanto, uma análise de sensibilidade pode ser realizada. Conforme mencionado anteriormente, os modelos de calibragem, assim como o AMLM, exigem informações sobre duas elasticidades, que podem vir de diferentes fontes (estudos, relatórios e opiniões, por exemplo) e que podem, portanto, apresentar alguma variação entre as diversas fontes ou até mesmo erros de avaliação ou da estimativa. Por construção o AMLM depende fortemente da confiança que o analista possui sobre as elasticidades. Desta forma, 
apresentam-se abaixo os resultados da variação das elasticidades para as combinações entre os elementos do conjunto $\{-0,5 ;-1 ;-1,5\}$ de a elasticidades da indústria e o conjunto $\{-2 ;-2,5$; 3 \} de elasticidade-preço da TAM e seleciono preço médio (Tabela 9) e o maior aumento de preços (Tabela 10) como resultados representativos da simulação de fusão entre GOL e WEBJET.

Tabela 9 - Análise de Sensibilidade - Aumento no Preço Médio- Fusão GOL-WEBJET

\begin{tabular}{lccc}
\hline & \multicolumn{3}{c}{ Elast. Própria } \\
\hline Elast. da Ind. & -2 & -2.5 & -3 \\
-0.5 & 3,17 & 2,59 & 2,20 \\
-1 & $2,83^{*}$ & 2,41 & 2,10 \\
-1.5 & 1,71 & 1,77 & 1,71 \\
\hline
\end{tabular}

*Modelo de Referência, valor já calculado anteriormente.

Fonte: Elaboração do Autor

Tabela 10 - Análise de Sensibilidade - Maior Aumento de Preços- Fusão GOL-WEBJET

\begin{tabular}{lccc}
\hline \multicolumn{3}{c}{ Elast. Própria } \\
\hline Elast. da Ind. & -2 & -2.5 & -3 \\
-0.5 & 35,89 & 26,18 & 19,88 \\
-1 & $27,37 *$ & 21,86 & 17,70 \\
-1.5 & 14,10 & 14,64 & 14,00 \\
\hline \multicolumn{2}{l}{ *Modelo de Referência, valor já calculado anteriormente. } \\
Fonte: Elaboração do Autor
\end{tabular}

Em todas as oito simulações realizadas na análise de sensibilidade, os resultados revelaram que a WEBJET, assim como no modelo de referência com elasticidade da indústria -1 e elasticidade da TAM -2, foi a marca que apresentou a maior elevação de preços no mercado. Outros resultados também se mostraram robustos em relação à variação nos valores das elasticidades. De fato, em todos casos observa-se um aumento elevado de preços da WEBJET e aumentos moderados no preço médio. Por exemplo, para a combinação de elasticidades -1.5 (elasticidade agregada) e -2.5 (elasticidade da TAM), a WEBJET eleva preços em 14,64\% e o preço médio aumento em apenas $1,77 \%$. 
Ao contrário do intervalo de variação do preço médio [1,71;3,17], verificado na Tabela 9, o intervalo que compreende os aumentos de preços da WEBJET (Tabela 10) revela-se bem superior [14,00;35,89]. No entanto, a conclusão de que a operação gera significativas preocupações anticoncorrenciais se mostra é robusta à variação de valores das elasticidades, visto que mesmo no melhor cenário possível para as partes envolvidas na fusão, uma das marcas apresenta considerável aumento $(14,00 \%)$.

\section{Conclusão}

O Modelo Antitruste Logit (ALM), desenvolvido por Werden e Froeb (1994), tem servido de referência para previsão dos efeitos (anti) competitivos decorrentes de fusões horizontais em mercados com produtos diferenciados. O ALM não exige muitos dados, é rápido de computar e só necessita de informações sobre preços, fatias de mercado e duas elasticidades. Estes atributos fazem desta metodologia de calibração instrumento particularmente atraente para as analistas de agências antitruste e outros profissionais envolvidos na análise de concentrações horizontais, devido às restrições de tempo que normalmente enfrentam. No entanto, é notório que o modelo de demanda Logit impõe severas restrições sobre as elasticidades, que constituem importantes parâmetros econômicos na avaliação dos efeitos de uma fusão.

Este artigo apresenta o Antitrust Mixed Logit Model (AMLM), uma nova metodologia, cuja principal contribuição, que o distingue do ALM, é mostrar como calibrar o parâmetro de uma demanda Mixed Logit. Após determinados os parâmetros da demanda, o modelo segue a maioria dos modelos de simulação de fusão encontrados na literatura de organização industrial, ou seja, assume competição de Bertrand a fim de obter os custos marginais e simular aumentos de preços.

A grande vantagem do AMLM sobre a versão Logit (ALM) é a flexibilidade da demanda do Mixed Logit, que gera padrões mais razoáveis de substituição entre os bens e, conseqüentemente, previsões mais plausíveis sobre os efeitos de uma fusão. Além disso, ao contrário das abordagens econométricas para estimar os parâmetros de uma demanda Mixed Logit (Berry, Levinsohn e Pakes de 1995, e Nevo, 2001), o AMLM exibe atributos que são particularmente atraentes para agências de Antitruste: baixa exigência de dados e alta velocidade computacional. 


\section{Referências}

Berry, S. (1994) Estimating Discrete-Choice Models of Product Differentiation. Rand Journal of Economics, vol. 25, n. 2, pp. 242-262.

Berry, S., Levinsohn, J. e Pakes, A. (1995) Automobile Prices in Market Equilibrium. Econometrica, vol. 63 , n. 4 , pp. 841-890.

Budzinski, O. e Ruhmer, I. (2009) Merger simulation in competition policy: a survey. Journal of Competition Law \& Economics, vol. 6, n. 2, pp. 277-319.

Epstein, R. J. e Rubinfeld, D. L. (2002) Merger Simulation: A Simplified Approach with New Applications. Antitrust Law Journal, vol. 69, pp. 883-919.

Epstein, R. J. e Rubinfeld, D. L. (2004) Merger Simulation with Brand-Level Margin Data: Extending PCAIDS with Nests. Advances in Economic Analysis \& Policy, vol. 4, n. 1, p.1.

Ferreira, N. S. (2011) Estudo empírico de práticas predatórias de companhias aéreas. Journal of Transport Literature, vol. 5, n. 3, pp. 89-122.

McFadden, D. (1974) Conditional Logit Analysis of Qualitative Choice Behavior. In: Zarembka, P., (ed.) Frontiers in Econometrics. New York: Academic Press.

Nevo, A. (2000) Mergers with Differentiated Products: The Case of the Ready-to-Eat Cereal Industry, Rand Journal of Economics, vol. 31, pp. 395-421.

Nevo, A. (2001) Measuring Market Power in the Ready-to-Eat Cereal Industry, Econometrica, vol. 69, n. 2, pp.307-342.

Petrin, A. (2002) Quantifying the benefits of New Products: The Case of the Minivan. Journal of Political Economy, vol. 110, n. 4, pp. 705-729.

Vassallo, M. D. (2010) Simulação de Fusão com Variações de Qualidade no Produto das Firmas: Aplicação para o Caso do Code-Share Varig-TAM. Journal of Transport Literature, vol. 4, n. 2, pp. 50-100.

Werden, G. J. e Froeb L. M. (1994) The Effects of Mergers in Differentiated Products Industries: Logit Demand and Merger Policy. Journal of Law, Economics, \& Organization, vol. 10, pp. 407-426.

Werden, G. J. e Froeb L. M. (2008) Unilateral Competitive Effects of Horizontal Mergers. In: Buccirossi, P. (ed.) Handbook of Antitrust Economics. Cambridge: MIT Press. 